\title{
Cost-Effectiveness of Real-World Administration of Concomitant Viscum album L. Therapy for the Treatment of Stage IV Pancreatic Cancer
}

\author{
Anja Thronicke $\mathbb{D}^{1},{ }^{1}$ Thomas Reinhold ${ }^{2}{ }^{2}$ Philipp von Trott, ${ }^{3}$ Harald Matthes $\mathbb{D D}^{1,3,4}$ \\ and Friedemann Schad $\mathbb{D D}^{1,4}$ \\ ${ }^{1}$ Research Institute Havelhöhe, Kladower Damm 221, 14089 Berlin, Germany \\ ${ }^{2}$ Institute of Social Medicine, Epidemiology and Health Economics, Charité-Universitätsmedizin Berlin, \\ Corporate Member of Freie Universität Berlin, Humboldt-Universität zu Berlin, Berlin Institute of Health, \\ 10117 Berlin, Germany \\ ${ }^{3}$ Oncological and Palliative Care Centre, Hospital Gemeinschaftskrankenhaus Havelhöhe, Kladower Damm 221, \\ 14089 Berlin, Germany \\ ${ }^{4}$ Medical Clinic for Gastroenterology, Infectiology and Rheumatology CBF, \\ Institute of Social Medicine, Epidemiology and Health Economics, Charité-Universitätsmedizin Berlin, \\ Corporate Member of Freie Universität Berlin, Humboldt-Universität zu Berlin, Berlin Institute of Health, \\ 10117 Berlin, Germany
}

Correspondence should be addressed to Friedemann Schad; fschad@havelhoehe.de

Received 27 August 2019; Accepted 14 February 2020; Published 13 March 2020

Academic Editor: Víctor López

Copyright (๑) 2020 Anja Thronicke et al. This is an open access article distributed under the Creative Commons Attribution License, which permits unrestricted use, distribution, and reproduction in any medium, provided the original work is properly cited.

Background. For patients receiving add-on Viscum album L. (VA) treatments for late-stage pancreatic cancer, an improved overall survival (OS) was observed. Only limited information regarding cost-effectiveness (CE) for comparisons between standard of care and standard of care plus add-on VA in stage IV pancreatic cancer treatment is available. The present study assessed the costs and cost-effectiveness of standard of care plus VA (V) compared to standard of care alone (C) for a hospital in Germany. Methods. An observational study was conducted using data from the Network Oncology clinical registry. Patients included had stage IV pancreatic cancer at diagnosis and received $C$ or $V$ treatment in a certified German Cancer Center. Cost and cost-effectiveness analyses (CEA) including the analysis of the incremental cost-effectiveness ratios (ICER) were performed from the hospital's perspective based on routine data from the financial controlling department and observed data on OS. The primary result of the analysis was tested for robustness in a bootstrap-based sensitivity analysis. Results. 88 patients $(C: n=34 ; V: n=54)$ were included, with a mean age 65.6 years and proportion of male patients $48.9 \%$. Adjusted hospital's total mean costs for patients from the $C$ and $V$ groups were $€ 10068.97$ (over an adjusted mean OS time of 7.1 months) and $€ 12267.94$ (over an adjusted mean OS time of 10.6 months), respectively. As to CEA analysis, a relevant total hospital's savings of $€ 260.81 /$ month mean OS for $V$ patients compared to $C$ was calculated. The costs per additional OS month gained (ICER) with the $V$ treatment compared to $C$ were $€ 628.28$. Furthermore, a relevant hospital savings of $€ 915.77$ per mean hospital stay and additional costs of $€ 75.09$ per mean hospitalization day in the $V$ group were observed compared to C. Conclusion. Based on this CEA analysis, from the hospital's point of view, the costs per mean month of OS and per mean hospital stay were lower for patients under combinational standard of care plus VA compared to patients receiving standard of care alone for the treatment of stage IV pancreatic cancer. Further prospective costeffectiveness studies are mandatory to reevaluate our findings. 


\section{Introduction}

Integrative oncology (IO) has been enormously developed and internationally established during the last decades in academic and public cancer centres [1]. Eight of 10 longterm survivors have already utilized IO therapies [2]. As per definition, IO "is a patient-centered, evidence-informed field of cancer care that utilizes mind and body practices, natural products, and/or lifestyle modifications from different traditions alongside conventional cancer treatments. Integrative oncology aims to optimize health, quality of life, and clinical outcomes across the cancer care continuum and to empower people to prevent cancer and become active participants before, during, and beyond cancer treatment" [3]. The American Society of Clinical Oncology (ASCO) has recently acknowledged the International Society of Integrative Oncology (SIO) guideline reflecting IO's impact on the improvement of health-related quality of life [4]. Nevertheless, the literature on cost-effectiveness (CE) of IO interventions is limited [5]. Thus, there is a critical need to keep investigating for which patients various IO models might be clinically effective and efficient $[5,6]$ as detailed analyses of variables determining increased healthcare expenditures are important for hospital systems and decision makers. IO concepts including add-on mistletoe (Viscum album Loranthaceae, VA) have been utilized with positive results, usually with high external validity, low adverse events, and high patient satisfaction [7]. Add-on VA treatments for latestage pancreatic cancer have recently been shown to improve OS [8]. In our recent analysis, we have also reported on advanced and metastasized patient's improved OS receiving IO concepts including add-on VA extracts [9]. Pancreatic cancer ranks fifth position in cancer-related deaths worldwide [10] and fourth position in Germany with a five-year survival rate of only of 9-10\% [11]. The majority (approx. $60 \%$ ) of the patients is diagnosed at an advanced stage [12]. Pancreatic resection with adjuvant chemotherapy (CTx) is the gold-standard approach [13], but in only about $20 \%$ of all cases, the tumour is resectable at diagnosis [14]. For palliative treatment, chemotherapy has proven to be superior to best supportive care only [15]. In regards to metastatic pancreatic cancer (mPC), several cost or cost-effective analyses have been conducted, and most of them focussed on specific procedures such as costs of screening methods [16] and standard therapies including resection, radiotherapy, and systemic therapies [17-19]. Cost-effectiveness (CE) analyses are economical evaluations of costs in monetary units (e.g., hospital's costs) and of effectiveness outcomes in nonmonetary units (e.g., saved life years or subjective health-related quality of life). As limited information regarding $\mathrm{CE}$ comparisons between standard of care and IO concepts including add-on VA is available and considering the recent efficacy data for add-on VA therapy for $\mathrm{mPC}$, there was an interest in the cost-effectiveness of the combinational therapy compared with the standard of care treatment. Thus, the purpose of the present analysis was to estimate the CE of combinational standard plus add-on VA compared with standard therapy alone in $\mathrm{MPC}$ patients from a hospital's perspective.

\section{Materials and Methods}

2.1. Study Design, Patients, and Primary Objective. A controlled nonrandomized observational monocentric cohort study was conducted revealing real-world data (RWD) [20] by analysing data from the Network Oncology (NO) clinical registry. The NO is a conjoint clinical register of hospitals, practitioners, and outpatient centres [21]. Patients were included in the analysis who were 18 years or older and who gave written consent, with a histologically proven primary diagnosis of stage IV pancreatic cancer receiving standard of care and surviving more than 21 days. Patients were not included when the death date or the last contact date was not available. Follow-up was performed routinely six months after first diagnosis and annually during the next years. Loss to follow-up was defined as no follow-up visits. The CE analysis took the perspective of the hospital Gemeinschaftskrankenhaus Havelhöhe Berlin (GKHB), at which the patients were treated and which is an Anthroposophic-integrative working hospital harbouring three German Cancer Society (DKG, Deutsche Krebsgesellschaft) certified Organ Centres as a DKG-certified Cancer Centre. The study served as a feasibility study for subsequent IO cost-effectiveness studies. The primary objective of this analysis was to evaluate the CE of VA in addition to standard of care compared to standard of care alone in stage IV pancreatic cancer patients from the hospital's perspective.

\subsection{Ethics Approval and Consent to Participate. The NO} study has been approved by the Ethics Committee of the Medical Association Berlin (Berlin-Ethik-Kommission der Ärztekammer Berlin). The reference number is Eth-27/10. Written informed consent has been obtained from all patients prior to study enrolment. The study complies with the principles laid down in the Declaration of Helsinki.

2.3. Data Collection. Structured queries from patient records were run for pancreatic cancer patients (International Classification of Diseases code: C25) using the clinical database NO. Demographic data and hospital-related data (diagnosis, pretreatment, and treatment) were retrieved from the NO. In addition, recorded TNM stages and/or documented metastases were queried with their according date and translated into the Union for International Cancer Control (UICC) stages according to the 8th edition of TNM Classification of Malignant Tumours. UICC stage at first diagnosis was defined as the earliest recorded stage within a month of the diagnosis date. Furthermore, standard oncological treatments were queried with their according date. Application of VA extracts in the context of an IO setting was retrieved with the start and end dates, the application type, and the pharmaceutical used. VA therapy was defined as lasting equal or more than four weeks. Information on hospital stays, length of each hospitalization, and cost weights for each patient indicating disease severity was retrieved from the GKHB hospital's cost-accounting database at the financial controlling department. Any inpatient visit included in the analysis was related to the diagnosis or treatment of the disease. For overall survival 
outcome analyses, patient's last record including date of death, the last documentation of personal contact, date of interdisciplinary tumour board conferences, or follow-up data were retrieved from the NO registry. Since the cost analyses should reflect the hospital's perspective, inpatient longitudinal cost data were obtained from the GKHB hospital's cost-accounting database according to the German Institute for Hospital Fee Systems (InEK) reporting principles. Cost data included the cost of primary therapy for pancreatic cancer, medication, hospital charges for surgery, anaesthesia, diagnostics, laboratories, professional fees, imaging, normal and intensive care units, and medical and nonmedical infrastructure as incurred. Outpatient costs were not included because they are not captured routinely in the data.

2.4. Allocation of Groups. Stage IV pancreatic patients included in the study were classified into one of the two groups: (a) $C$ group-patients received only standard of care and no add-on VA therapy and (b) $V$ group-patients that received standard of care and add-on VA therapy. Standard of care and add-on VA were applied as per routine clinical care. Nonrandomized allocation to the treatment groups was performed by the physician after elaborate information and patient's decision on treatment options. Applied VA preparations included Abnobaviscum, Helixor, and Iscador VA extracts and were given subcutaneously according to the SmPC [22-24]. Off-label VA application (intravenous and intratumoural) was performed in individual cases.

2.5. Statistical Analyses. All statistical analyses were conducted using the software R, version 3.5.2 (2018-12-20 [42]) with the exception of sensitivity analysis which was performed by using MS Excel 2016.

Data are presented using descriptive statistics, normally distributed continuous data by the mean and standard deviation (SD) or 95\% CI, and skewed distributions by the median and 95\% confidence interval (CI). Binary and categorical variables were presented as absolute and relative frequencies using counts and percentages. For comparison of continuous variables between groups at baseline, the unpaired Student's $t$-test for independent samples was used. For comparison of categorical baseline variables, chi-square analyses were performed. All tests were performed twosided. $p$ values $<0.05$ were considered as significant.

For the survival outcome, Kaplan-Meier survival and multivariate stratified Cox proportional hazard analyses were calculated as previously reported [25]. The start date for survival analysis was the date of first diagnosis of stage IV pancreatic cancer. Patient survival was calculated from the index date to the patient's last record. Respective data for patient's last record were retrieved from the NO registry. Kaplan-Meier survival was calculated for both groups. For survival analysis including Kaplan-Meier curves and rightcensored time-to-event analyses, as well as univariate and multivariate Cox proportional hazard models, the R-package "survival" version 2.41-3 was used, for the implementation of nonparametric estimators for censored event history analysis, the package "prodlim," version 1.6.1 was used, and to draw survival curves, the package "survminer," version 0.4.0 was used. Age adjustment of mean OS was performed for both groups using the $\operatorname{coxph}(\operatorname{surv}())$ function from the package survival $(\mathrm{R})$. The surv function creates a survival object, which is usually used as a response variable in a model formula. The coxph function fits a Cox proportional hazards regression model incorporating time-dependent variables (here: time as a single survival time/follow-up time value with status indicators: $1=$ dead and $0=$ alive for possible censoring), time-dependent strata (here: group), and other extensions $[26,27]$. To analyse how different factors influence the hazard on patient survival and to reduce potential confounding bias, we employed multivariate stratified Cox proportional hazard model adjusting for age, gender, BMI, comorbidities, and oncological treatment variables including add-on VA treatment. Prior to this analysis, verification analyses were performed whether or not proportional hazard assumptions were met.

Mean number of hospital stays, mean hospitalization length in days, and mean cumulative hospital costs (in $€$ ) were calculated for each treatment group and adjusted for significant baseline differences (here: age) using the anov$\mathrm{a}(\operatorname{lm}())$ function in $\mathrm{R}$ to fit a linear model and to carry out analysis of covariance. Cost-associated factors were additionally analysed using multivariable linear regression.

2.6. Cost-Effectiveness Analyses. For measuring the cost-effectiveness, data on mean OS, mean number of hospital stays, and results on mean hospitalization length were combined with the results on mean total hospital costs. As a result, the mean hospital costs per month mean OS, the mean hospital costs per mean hospital stay, and the mean hospital costs per mean period of hospitalization were reported.

In the case where the intervention would show a better effect in terms of OS and would be more expensive than the control, the incremental cost-effectiveness ratio (ICER) was calculated. The ICER is defined as a ratio of an additional cost-effectiveness of one intervention (here, $V$ ) compared to another intervention (here, $C$ ) and was calculated through dividing the group cost difference by effectiveness difference translating into the mathematical equation ((mean hospital costs $V$ - mean hospital costs $C)$ / (mean months OS $V-$ mean months OS C)). To determine to what extent the primary cost-effectiveness results may vary due to many replications, a bootstrap analysis with random 1000-fold resampling population was performed. This analysis accounted for the heterogeneity of hospital's resource consumptions observed in the study. The results of the bootstrap samples were plotted into the fourquadrant diagram (cost-effectiveness plane), which gives graphical information on the results' robustness.

\section{Results}

3.1. Patients. From 98 patients who were screened, 10 patients were not included due to missing data, see Figure 1. Thus, 88 patients ( $C: n=34 ; V: n=54$ ) were included for 


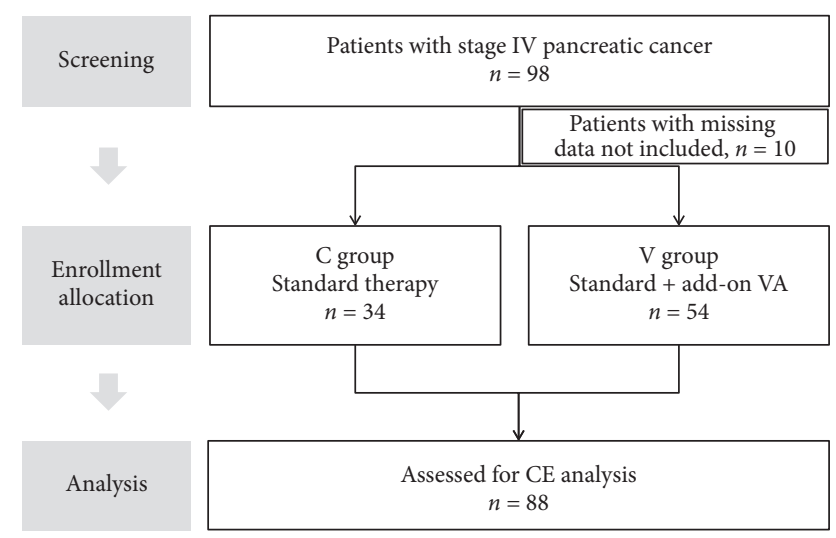

FIGURE 1: Flowchart of the study population. CE, cost-effectiveness; VA, Viscum album L., mistletoe.

subsequent outcome analysis. Patient's demographic and clinical characteristics are shown in Table 1. The mean age of the patients was 65.6 years. Patients in the $V$ group were on average 4.9 years younger than patients in the $C$ group $(C$ : 68.6 years; $V: 63.7$ years; $p=0.04) .48 .9 \%$ of the patients were male. As to gender, BMI, occurrence of comorbidities, arterial hypertension, diabetes mellitus type II, chronic pancreatitis, and pancreatic insufficiency, the patients seem to be largely balanced.

With respect to the total cohort, guideline-oriented surgery $(20.5 \%)$ and oncological treatment $(56.8 \%$ gemcitabine as first-line CTx) were applied. In the $V$ group, add-on Viscum album applications were applied with the majority having received add-on $\mathrm{VA}$ from the apple tree $(66.7 \% \mathrm{VA}$ mali), from the ash tree (57.4\% VA fraxini), from the oak tree (44.4\% VA quercus), and from the fir tree $(24.1 \% \mathrm{VA}$ abietis), see Table 2 .

3.2. Overall Survival. When patients with $V$ treatment are compared to those with $C$ treatment, the OS showed a significant increase. Age-adjusted median OS for $C$ and $V$ was 5.63 (95\% CI: 3.37-8.63) and 8.43 months (95\% CI: 6.5-12.47), respectively, indicating that patients from the $V$ group lived 2.8 months longer than patients from the $C$ group $(\chi 2=7, p=0.008)$. The age-adjusted mean OS for $C$ and $V$ was 7.1 months and 10.6 months, respectively, and the difference between both groups was 3.5 months, see Table 3 . As no death occurred in both groups before the start of the treatment and as time until treatment was not significantly different between both groups $(p=0.15)$, a time-dependent bias could be precluded. To analyse how different factors influence the hazard of patient OS and to reduce potential confounding bias, the multivariate stratified Cox proportional hazard model adjusting for age, gender, BMI, comorbidities, and oncological treatment resulted in an adjusted hazard risk (aHR) of 0.35 for chemotherapy indicating a significant $65 \%$ reduced risk for the risk of death (aHR: 0.35 , 95\% CI: $0.20-0.62, p=0.0004$ ), while the adjusted hazard risk (aHR) for additional VA therapy was 0.47 indicating a significant $53 \%$ reduced hazard of death (aHR: 0.47, 95\% CI $=0.28-0.80, p=0.005)$. Furthermore, the direction of impact on hazard of death was negative (i.e., positive for OS) for radiation and surgery but not significant, data not shown. Finally, comorbidities significantly increased the hazard of death by factor 2.1 (aHR: 2.09, 95\% CI: 1.13-3.87, $p=0.02$ ).

3.3. Cost Outcomes. The cost estimates for both treatment groups are shown in Table 3. Age-adjusted mean hospital's costs were $€ 10069$ for the $C$ group and $€ 12268$ for the $V$ group, see Table 3 . Highest hospital's expenditures per patient in the $C$ group were documented for the normal ward, followed by expenditures for endoscopic diagnostics and therapy, general diagnostics, and surgery, while in the $V$ group, the highest expenditures were for the normal ward, followed by expenditures for diagnostics, radiology, and for endoscopic diagnostics and therapy, see Figure 2. Multivariable regression analysis revealed that hospitals costs were highly statistically negatively associated with radiation therapy (estimate -0.25 ; $p=0.009$ ). Furthermore, surgery was negatively but not statistically significantly associated with hospital's costs, while the direction of association with age, add-on VA treatment, comorbidities, and chemotherapy was positive but not statistically significant, data not shown.

3.4. Cost-Effectiveness. The survival analyses demonstrated that patients in the $V$ group showed longer survival than those receiving standard of care (a 3.5 month longer overall mean survival). Cost analyses have shown that this longer survival was associated with additional hospital costs (Table 3). After combining survival data and total costs, the mean costs per mean month OS for the $C$ and $V$ groups were $€ 1418.16$ and $€ 1157.35$, respectively. Compared to $C$, patients with $V$ treatment had relevant hospital's savings of $€ 260.81$ per mean OS, see Table 3. This translates into annual hospital's savings of $€ 3129.72$.

We calculated an ICER of $€ 628.28$ per additional month OS resembling the costs for the improvement of one OS month gained with the $V$ treatment compared to the $C$ treatment. This would translate into $€ 7539.32$ per additional year OS indicating the costs of improvement of one OS year gained with the new treatment compared to standard of care. Further analyses revealed that (driven by longer survival) patients from the $V$ group had on average 1.2 more hospital stays since first diagnosis than patients from the $C$ group, see Table 3. Adjusted mean hospital visits in the $C$ - and in the $V$-group were 2.21 and 3.37 visits, respectively. As to costeffectiveness, the mean costs per mean hospital stay for the $C$ and $V$ patients were $€ 4556.10$ and $€ 3640.33$, respectively. Consequently, a relevant hospital's saving of $€ 915.77$ per mean hospital stay of patients in the $V$ group compared to $C$ was calculated, see Table 3. Patients from the $V$ group had on average a 1.43 day longer hospital stay than $C$ treatment patients: the adjusted mean duration of stays for patients from the $V$ group was 13.9 days compared to 12.47 days in the $C$ group, see Table 3 . The calculated mean costs per mean hospitalization day for $C$ and $V$ were $€ 807.50$ and $€ 882.59$, respectively, see Table 3. An independent analysis revealed that the median DRG cost weights in the $C$ and $V$ groups 
TABLE 1: Baseline characteristics of included patients with stage IV mPC.

\begin{tabular}{|c|c|c|c|c|}
\hline Variable & All $(n=88)$ & $C(n=34)$ & $V(n=54)$ & $p$ value \\
\hline Age, mean, SD & $65.60(11.40)$ & $68.61(10.06)$ & $63.71(11.87)$ & 0.04 \\
\hline Gender, female, $n(\%)$ & $45(51.1)$ & $17(50.0)$ & $28(51.90)$ & 1 \\
\hline Body mass index $<18.5$, underweight, $n(\%)$ & $56(93.3)$ & $20(90.9)$ & $36(94.70)$ & 0.97 \\
\hline Comorbidities, yes, $n(\%)$ & $59(67.0)$ & $21(61.8)$ & $38(70.40)$ & 0.55 \\
\hline Arterial hypertension, $n(\%)$ & $32(36.4)$ & $16(47.1)$ & $16(29.6)$ & 0.10 \\
\hline Diabetes mellitus type II, $n(\%)$ & $13(14.8)$ & $6(17.6)$ & $7(13.0)$ & 0.55 \\
\hline Chronic pancreatitis, $n(\%)$ & $2(2.3)$ & $2(5.9)$ & 0 & 0.15 \\
\hline Pancreatic insufficiency, $n(\%)$ & $1(1.1)$ & $1(2.9)$ & 0 & 0.39 \\
\hline
\end{tabular}

$n$, number of patients; \%, percent; $\mathrm{SD}$, standard deviation.

TABLE 2: Composition of add-on VA treatment in the $V$ group.

\begin{tabular}{lc}
\hline & $N(\%)$ \\
\hline VA abietis & $13(24.1)$ \\
VA aceris & $2(3.7)$ \\
VA fraxini & $31(57.4)$ \\
VA mali & $36(66.7)$ \\
VA pini & $5(9.3)$ \\
VA quercus & $24(44.4)$ \\
\hline
\end{tabular}

Number of $V$ group patients ( $n=54$ ) exposed to various add-on mistletoe (VA) extracts pooled per host tree. $n=$ number of patients; the total number of patients per VA remedy does not necessarily add to $100 \%$ as patients may have received various combinations of VA treatment; $N=$ number of patients; \%, percent.

were similar between both groups ( $C: 1.10 ; V: 1.14)$ indicating similar disease severities, data not shown.

For the outcome mean costs per patient from the hospital perspective in combination with mean month OS, a probabilistic sensitivity analysis was performed. Figure 3 shows a scatter plot of all replicated results for bootstrap sensitivity analyses. Most single dots are located in the upper right-hand quadrant indicating a probability of $87.6 \%$ and confirming the robustness of the CEA outcome. Nevertheless, a small proportion of single dots located in the lower right-hand quadrant points to a $12.4 \%$ probability of hospital's cost savings.

\section{Discussion}

To our knowledge, this is the first study to directly analyse costs and cost-effectiveness of standard of care plus add-on mistletoe therapy in patients with stage IV pancreatic cancer using patient-level data and actual hospital costing in Germany. From the hospital's point of view, our analysis revealed that compared to standard of care $(C)$, patients treated with the combinational therapy $(V)$ received a costeffective therapy with relevant total hospital's savings per mean month OS and per mean hospital stay.

In the present study, we could show that CTx was positively associated with good survival outcome revealing the clinical gold standard for this cancer stage and correlating with data from published studies with CTx's superiority as to OS for advanced or unresectable pancreatic cancer $[15,28]$. Next, patients receiving the additional treatment with VA in the present study showed a longer median and mean OS and a significant association with a positive survival outcome. This resembles published clinical outcome results as shown in an RCT and in a real-world analysis for advanced and metastatic pancreatic cancer patients $[8,9]$. A relevant negative association of comorbidities including diabetes mellitus type II and chronic pancreatitis with survival outcome was observed in our patients and correlates with other published data [11]. Cost analyses revealed that radiation positively influenced hospital's costs which may be explained by subsequent patient's improvement of tumour status and less hospital's expenditures with treatment efforts. Further cost analyses in the present study indicated somewhat higher costs of the combinational treatment in favourable relation to a better outcome (OS). Higher inpatient hospital costs could either derive from an increased number of hospital stays due to longer OS, from longer hospitalization, from higher DRG cost weights (as an indicator for disease severities), or from a combination of these variables. An independent analysis in the present study revealed that the DRG cost weights were similar between both groups indicating similar and balanced disease severities. Thus, the higher mean costs in the group with the combinational treatment are not explainable by disease severity. Rather, a combination of the slightly higher number of hospital stays due to longer survival, as shown here for the $V$ treatment, would serve as an explanation. It has been reported that the coincidence of complementary treatment with increased care durations does not have to be necessarily correlated to a more severe disease status of patients [29]. Given a simultaneous better effectiveness in the present study, combinational therapy with add-on VA has been shown to be noninferior to standard of care alone in terms of cost-effectiveness revealing hospital's savings for this treatment compared to the control. Furthermore, hospital's savings per patient's hospital stay for patients from the $V$ group compared to the $C$ group were observed, indicating good CEA outcome results. On the contrary, hospitals expenditures per day of each hospitalization were higher in the $V$ group compared to the $C$ group. This can be explained by the application of several Anthroposophic add-on complementary therapies (beyond add-on VA therapy) $[29,30]$. However, compared to earlier studies on pancreatic carcinoma, our data indicate less visits and shorter hospitalization time of the total observed $\mathrm{mPC}$ cohort in our study, reflecting current real-life treatment of pancreatic cancer patients as they lie shorter and most of the preparations are increasingly performed in an ambulant setting [31]. Therefore, during further prospective cost-effectiveness 
TABLe 3: Age-adjusted costs and cost-effectiveness measures from hospital's perspective.

\begin{tabular}{|c|c|c|c|}
\hline & C & $V$ & $\Delta_{V-C}$ \\
\hline \multicolumn{4}{|l|}{ Costs } \\
\hline Hospital costs*, mean $€,(95 \% \mathrm{CI})$ & $10068.97(6659.87 ; 13478.06)$ & $12267.94(9593.67 ; 14942.20)$ & 2198.97 \\
\hline \multicolumn{4}{|l|}{ Outcomes } \\
\hline OS, median months, (95\% CI) & $5.63(3.37 ; 8.63)$ & $8.43(6.50 ; 12.47)$ & 2.8 \\
\hline OS, mean months*, $(95 \% \mathrm{CI})$ & $7.1(4.75 ; 9.45)$ & $10.6(8.44 ; 22.86)$ & 3.5 \\
\hline Hospital stays* ${ }^{*}$ mean number, $(95 \% \mathrm{CI})$ & $2.21(-0.59 ; 5.01)$ & $3.37(-1.78 ; 8.53)$ & 1.16 \\
\hline Hospitalization length*, mean days, (95\% CI) & $12.47(-0.59-5.01)$ & $13.90(-1.42 ; 29.23)$ & 1.43 \\
\hline \multicolumn{4}{|l|}{ CE outcomes } \\
\hline Costs per month OS*, mean $€ /$ mean months & 1418.16 & 1157.35 & -260.81 \\
\hline Costs per hospital stay*, mean $€ /$ mean number & 4556.10 & 3640.33 & -915.77 \\
\hline Costs per days of hospitalization*, mean $€ /$ mean days & 807.50 & 882.59 & 75.09 \\
\hline
\end{tabular}

Hospital's costs per mPC patient according to the treatment group; *adjusted for age; $€$, euro; CI, confidence interval; OS, overall survival; CE, costeffectiveness; $C, C$ group; $V, V$ group; $\Delta$, difference.

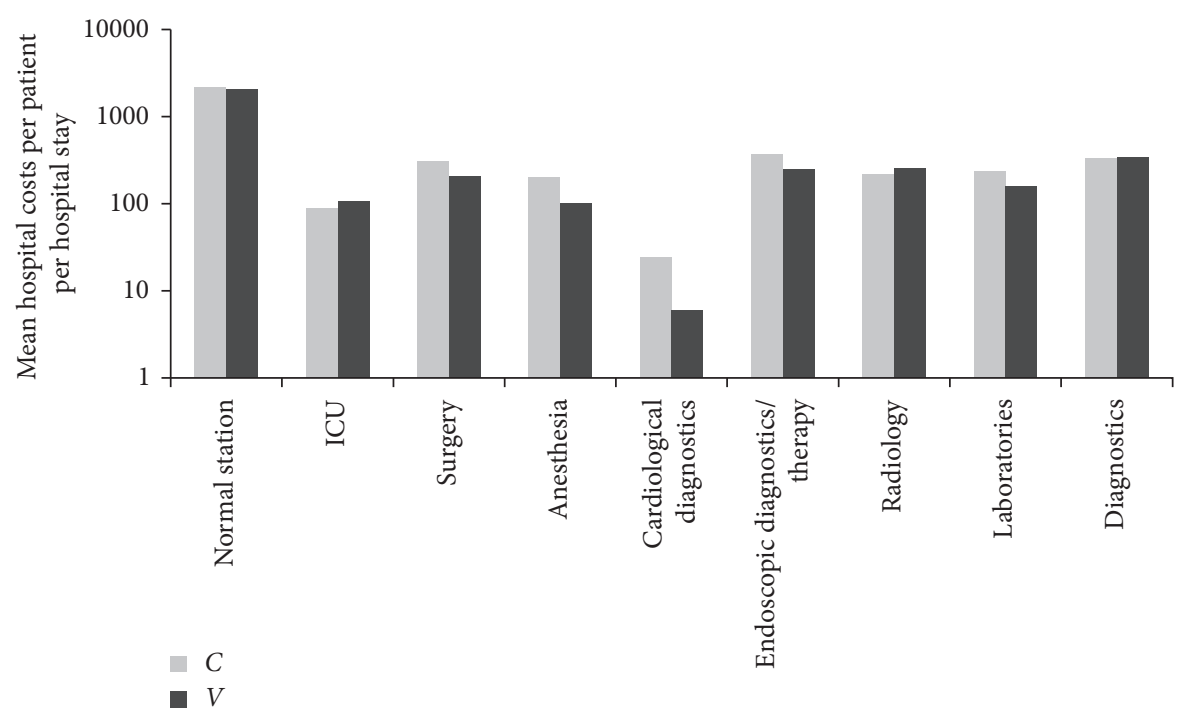

FIgUre 2: Mean hospital's costs per patient per hospital stay according to the treatment groups $C$ and $V$ in reliance with the German INEK cost categories, logarithmical y-scale; $€$.

studies and by including outpatient cost data in future projects, we will be able to draw a more comprehensive cost spectrum for mPCs. Even though health-related quality of life (HRQL) outcomes in the present study were not assessed, add-on VA's positive impact on HRQL has been repeatedly published [32]. Assuming $V$ treatment's noninferiority to $C$ treatment in terms of HRQL in $\mathrm{mPC}$ patients, hospital's costs of $€ 7539.32$ per one year overall survival gained (ICER) by the $V$ treatment compared to the $C$ treatment are rather low and would thus seem to be affordable in oncological settings.

Limitations of the present analysis include study's retrospective cost analysis, single institution nature, and the limited transferability of cost and CE data to other countries. In addition, through unblinded assignment of add-on VA, physicians could have unintentionally selected patients with better prognosis for this study arm. However, due to known and obvious local skin reactions caused by VA, the procurement of a blinded study seems almost not possible. Furthermore, it has been stated that patients with a healthier lifestyle may be more open for additional complementary therapies and could have selected add-on VA therapy. As sound lifestyle data were not available, this aspect cannot be ruled out so far. Generally, complementary treatment options are frequently enquired by patients in advanced and progressive cancer conditions that could rather result in negative selection bias in the addon treatment cohort. Further limitations may be the observational nature of the present study, and therefore our findings and conclusions have to be handled with caution. However real-world data as presented here may increasingly contribute to a circular model of evidence [20, 33], complementing published impact of add-on VA in oncology. In future, prospective cost-effectiveness studies with larger patient numbers through application of a multicentre design should limit potential bias. The strength of the present study is its pragmatic design under typical hospital conditions, the inclusion of real-life data, and the model's sensitivity analysis strengthening our observation that the results were comparably robust to the heterogeneity in the underlying patient sample. Thus, the external validity of our data may be high as patient's characteristics, 


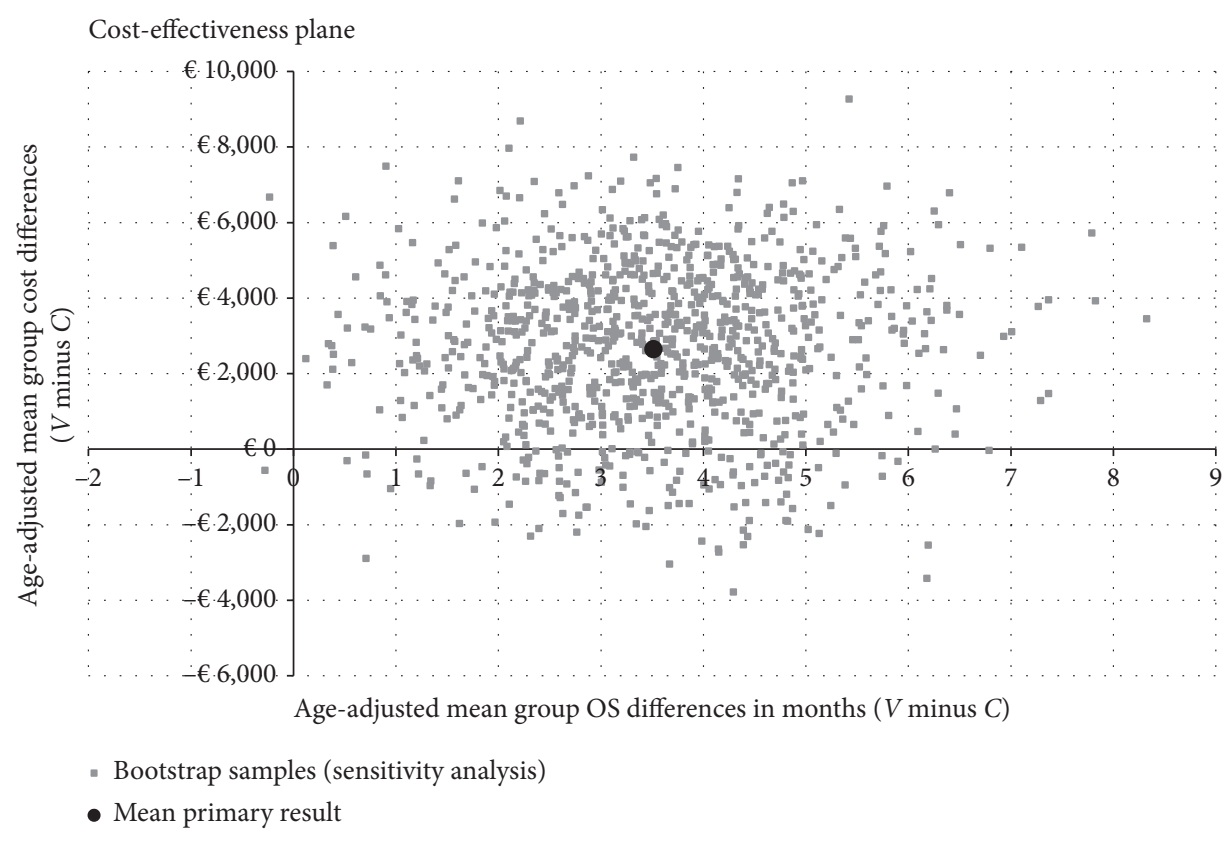

FIGURE 3: Sensitivity analyses of the outcome cost per month OS from the hospital's perspective. Incremental cost-effectiveness plane showing random 1000-fold resampled estimates (bootstrap analysis) of incremental costs and benefits (age-adjusted mean cost per patient in combination with age-adjusted mean month OS) of using $V$ treatment for mPC versus $C$ treatment.

types of treatments, and the effect outcome are compared to published data. This study may state a first onset for a discussion with healthcare administrators and policy makers on the cost-effectiveness of IO concepts for mPC inpatients in German Cancer Centres.

\section{Conclusions}

This is to our knowledge the first extensive evaluation of costs and cost-effectiveness associated with the add-on VA applications in $\mathrm{mPC}$ in a German hospital. Based on our findings, the combined standard of care plus VA therapy in these patients seems to be more effective than standard of care only and bears the potential to save hospital costs. In summary, the findings of the present study suggest that the inpatient costs for the combinational $V$ therapy compared to $C$ come in a favourable relation to a better outcome for stage IV pancreatic patients. Additionally, costs per mean month of OS and per mean hospital stay were lower for $V$ compared to $C$ treatment. The available data were of observational nature. Further prospective cost-effectiveness studies are mandatory to reevaluate our findings.

\section{Data Availability}

The anonymized data that support the findings of this study are openly available in the repository "figshare" (https:// figshare.com/articles/cea/8907338).

\section{Disclosure}

The funders had no role in study design, data collection and analysis, decision to publish, or preparation of the manuscript. By contract, researchers were independent from the funder.

\section{Conflicts of Interest}

FS reports grants from Helixor Heilmittel $\mathrm{GmbH}$, grants from Abnoba $\mathrm{GmbH}$, grants from Iscador $\mathrm{AG}$, and grants from AstraZeneca outside the submitted work. Grants from Helixor Heilmittel GmbH and AstraZeneca included travel costs and honoraria for speaking. There are no other relationships/conditions/circumstances that present a potential conflict of interest. There are no patents, products in development, or marketed products to declare. No payment was received for any other aspects of the submitted work. The other authors have declared that no conflicts of interest exist.

\section{Authors' Contributions}

AT, TR, PvT, HM, and FS conceptualized the study; AS curated the data; formal analysis/visualization was performed by AT and TR; funding acquisition was done by AT, HM, and FS; AT, TR, PvT, HM, and FS performed investigation; AT, TR, PvT, HM, and FS were involved in methodology; FS was responsible for project administration/ resources/software/supervision; AT, TR, PvT, HM, and FS performed validation; AT wrote the original draft; AT, TR, PvT, HM, and FS wrote, reviewed, and edited the final manuscript.

\section{Acknowledgments}

The authors would like to thank the financial controlling department at the GKHB and the staff members at the GKHB 
and FIH involved in the present work. The Network Oncology was funded by unrestricted research grants from Iscador AG Arlesheim, Switzerland; Abnoba GmbH Pforzheim, Germany; and Helixor GmbH Rosenfeld, Germany.

\section{References}

[1] H. Greenlee and Z. Shi, "Implementing integrative oncology: hopes and challenges," Journal of Oncology Practice, vol. 15, no. 1, pp. 17-18, 2019.

[2] G. M. John, D. L. Hershman, L. Falci, Z. Shi, W.-Y. Tsai, and H. Greenlee, "Complementary and alternative medicine use among US cancer survivors," Journal of Cancer Survivorship, vol. 10, no. 5, pp. 850-864, 2016.

[3] C. M. Witt, L. G. Balneaves, M. J. Cardoso et al., "A comprehensive definition for integrative oncology," JNCI Monographs, vol. 2017, no. 52, 2017.

[4] G. H. Lyman, H. Greenlee, K. Bohlke et al., "Integrative therapies during and after breast cancer treatment: ASCO endorsement of the SIO clinical practice guideline," Journal of Clinical Oncology, vol. 36, no. 25, pp. 2647-2655, 2018.

[5] K. I. Block, "Could integrative cancer treatment be cost-saving and resuscitate a submerged medical system?" Integrative Cancer Therapies, vol. 8, no. 3, pp. 205-207, 2009.

[6] T. Sundberg, L. Hussain-Alkhateeb, A. Inghilesi Larsson, D. Sibbritt, and T. Falkenberg, "Exploring sick leave in integrative care-retrospective observations and future study recommendations," The Journal of Alternative and Complementary Medicine, vol. 25, no. 4, pp. 377-384, 2019.

[7] G. S. Kienle, H. Kiene, and H. U. Albonico, Anthroposophic Medicine: Effectiveness, Utility, Costs, Safety, Schattauer Verlag, Stuttgart, Germany, 2006.

[8] W. Tröger, D. Galun, M. Reif, A. Schumann, N. Stanković, and M. Milićević, "Viscum album [L.] extract therapy in patients with locally advanced or metastatic pancreatic cancer: a randomised clinical trial on overall survival," European Journal of Cancer, vol. 49, no. 18, pp. 3788-3797, 2013.

[9] J. Axtner, M. Steele, M. Kröz, G. Spahn, H. Matthes, and F. Schad, "Health services research of integrative oncology in palliative care of patients with advanced pancreatic cancer," BMC Cancer, vol. 16, p. 579, 2016.

[10] R. L. Siegel, K. D. Miller, and A. Jemal, "Cancer statistics, 2017," CA: A Cancer Journal for Clinicians, vol. 67, no. 1, pp. 7-30, 2017.

[11] P. Kaatsch, C. Spix, A. Katalinic et al., Krebs in Deutschland für 2013/2014, Robert Koch-Institut und die Gesellschaft der Epidemiologischen Krebsregister in Deutschland, Berlin, Germany, 11th edition, 2017.

[12] M. Bardou and I. Le Ray, "Treatment of pancreatic cancer: a narrative review of cost-effectiveness studies," Best Practice \& Research Clinical Gastroenterology, vol. 27, no. 6, pp. 881-892, 2013.

[13] T. Seufferlein, J. B. Bachet, E. Van Cutsem, P. Rougier, and E. G. W. Group, "Pancreatic adenocarcinoma: ESMO-ESDO clinical practice guidelines for diagnosis, treatment and follow-up," Annals of Oncology, vol. 23, no. Suppl 7, pp. vii33vii40, 2012.

[14] A. Vincent, J. Herman, R. Schulick, R. H. Hruban, and M. Goggins, "Pancreatic cancer," The Lancet, vol. 378, no. 9791, pp. 607-620, 2011.

[15] D. Yip, C. Karapetis, A. Strickland, C. B. Steer, and D. Goldstein, "Chemotherapy and radiotherapy for inoperable advanced pancreatic cancer," The Cochrane Database of Systematic Reviews, no. 3, Article ID CD002093, 2006.
[16] S. J. Rulyak, M. B. Kimmey, D. L. Veenstra, and T. A. Brentnall, "Cost-effectiveness of pancreatic cancer screening in familial pancreatic cancer kindreds," Gastrointestinal Endoscopy, vol. 57, no. 1, pp. 23-29, 2003.

[17] V. C. Tam, Y. J. Ko, N. Mittmann et al., "Cost-effectiveness of systemic therapies for metastatic pancreatic cancer," Current Oncology, vol. 20, no. 2, pp. e90-e106, 2013.

[18] C. L. Attard, S. Brown, K. Alloul, and M. J. Moore, "Costeffectiveness of folfirinox for first-line treatment of metastatic pancreatic cancer," Current Oncology, vol. 21, no. 1, pp. e41-51, 2014.

[19] J. D. Murphy, D. T. Chang, J. Abelson et al., "Cost-effectiveness of modern radiotherapy techniques in locally advanced pancreatic cancer," Cancer, vol. 118, no. 4, pp. 1119-1129, 2012.

[20] S. Khozin, G. M. Blumenthal, and R. Pazdur, "Real-world data for clinical evidence generation in oncology," Journal of the National Cancer Institute, vol. 109, no. 11, 2017.

[21] F. Schad, J. Axtner, A. Happe et al., "Network oncology (NO) - a clinical cancer register for health services research and the evaluation of integrative therapeutic interventions in anthroposophic medicine," Forschende Komplementärmedizin/Research in Complementary Medicine, vol. 20, no. 5, pp. 353-360, 2013.

[22] Abnobaviscum-Information for Healthcare Professionals, Recommendations for Use.

[23] Fachinformation helixor," 2017, http://misteltherapie.at/diemisteltherapie/gebrauchs-fachinformation.

[24] Fachinformation iscador. 2012.

[25] F. Schad, A. Thronicke, M. L. Steele et al., "Overall survival of stage IV non-small cell lung cancer patients treated with Viscum album L. in addition to chemotherapy, a real-world observational multicenter analysis," PLoS One, vol. 13, no. 8, Article ID e0203058, 2018.

[26] P. K. Andersen and R. D. Gill, "Cox's regression model for counting processes, a large sample study," The Annals of Statistics, vol. 10, no. 4, pp. 1100-1120, 1982.

[27] T. Therneau and P. Grambsch, Modeling Survival Data: Extending the Cox Model, Springer-Verlag, Berlin, Germany, 2000.

[28] V. Chin, A. Nagrial, K. Sjoquist et al., "Chemotherapy and radiotherapy for advanced pancreatic cancer," The Cochrane Database of Systematic Reviews, vol. 3, no. 3, Article ID CD011044, 2018.

[29] J. Heinz, W. Fiori, P. Heusser, and T. Ostermann, "Cost analysis of integrative inpatient treatment based on DRG data: the example of anthroposophic medicine," Evidence-Based Complementary and Alternative Medicine, vol. 2013, no. 7, Article ID 748932, 2013.

[30] A. Thronicke, S. L. Oei, A. Merkle et al., "Integrative cancer care in a certified cancer centre of a German anthroposophic hospital," Complementary Therapies in Medicine, vol. 40, pp. 151-157, 2018.

[31] C. B. O’Neill, C. L. Atoria, E. M. O’Reilly, J. LaFemina, M. C. Henman, and E. B. Elkin, "Costs and trends in pancreatic cancer treatment," Cancer, vol. 118, no. 20, pp. 5132-5139, 2012.

[32] M. A. Horneber, G. Bueschel, R. Huber, K. Linde, and M. Rostock, "Mistletoe therapy in oncology," The Cochrane Database of Systematic Reviews, no. 2, Article ID CD003297, 2008.

[33] H. Walach, T. Falkenberg, V. Fønnebø, G. Lewith, and W. B. Jonas, "Circular instead of hierarchical: methodological principles for the evaluation of complex interventions," $B M C$ Medical Research Methodology, vol. 6, p. 29, 2006. 